\title{
A Novel Bridge Section Model Endowed with Actively Controlled Flap Arrays Mitigating Wind Impact
}

\author{
Maria Boberg, Glauco Feltrin and Alcherio Martinoli
}

\begin{abstract}
In this work, we present the SmartBridge, a novel bridge section model equipped with actively controlled arrays of flaps aiming at mitigating wind-induced vibrations of longspan bridges. The active model, as well as its support structure, is described in detail and the key design choices are motivated. Finally, the capabilities of the system and the active control of the bridge section model with moving flaps was validated by wind tunnel experiments. In spite of the relative simplicity of a, manually tuned, control law, the results are encouraging and show a significant damping of the pitch vibration of the deck.
\end{abstract}

\section{INTRODUCTION}

Long-span bridges are particularly vulnerable to wind loads, owing to their inherently low structural damping, low natural frequencies, and adjacent fundamental torsional and vertical mode frequencies. This leads to wind-induced instabilities, causing potential damage to the whole structure.

Most solutions for this problem deployed on real bridges consist of passive elements that reduce the aerodynamic requirements on the cross-section [1]. However, a passive solution cannot adapt to dynamic wind conditions. Active solutions could potentially lead to a more favorable performance/cost trade-off in the building and maintenance phases as well as new opportunities for improving bridge aesthetics.

One possible active solution is to install multiple mobile flaps along the bridge girder in order to alter its aerodynamic profile, enabling stabilizing forces on the structure, a concept illustrated in Fig. 1. Furthermore, the angular position of the adjustable flaps is controlled as a function of the wind field and/or the displacement of the structure whose dynamic state can be measured with an underlying sensor network.

Although several control strategies for flaps damping bridge deck oscillations, in particular the flutter phenomenon, have been investigated, only a handful of research groups have provided experimental validation of bridge flutter control. Kobayashi et al. [2] managed to increase the wind speed at which flutter occurs by $50 \%$ with actively controlled flaps installed above the bridge deck. Kobayashi et al. [3] followed up the work and also showed an efficient flutter control with flaps attached directly to the bridge deck, as in Fig. 1. Furthermore, Hansen et al. [4, 5] also designed an active

Maria Boberg and Alcherio Martinoli are with the Distributed Intelligent Systems and Algorithms Laboratory, School of Architecture, Civil and Environmental Engineering, École Polytechnique Fédérale de Lausanne, Switzerland. maria.boberg@epfl.ch and alcherio.martinolidepfl.ch

Maria Boberg and Glauco Feltrin are with the Structural Engineering Laboratory, Swiss Federal Laboratories for Materials Science and Technology, Duebendorf, Switzerland. glauco.feltrin@empa.ch

This work has been partially sponsored by the Swiss National Science Foundation under grant number 200021-149543.

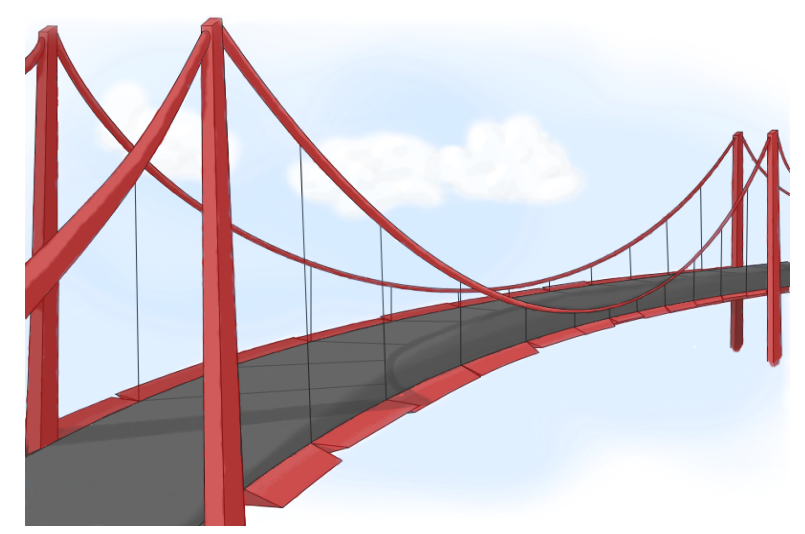

Fig. 1. Conceptual figure of the multi-flap wind mitigation strategy.

bridge deck capable of stabilizing the bridge vibrations, with actively controlled flaps attached to the deck.

Both Kobayashi et al. and Hansen et al. were actively controlling the flaps; however, there are also a few works investigating moving flaps controlled using passive mechanical elements. Wilde et al. [6] proposed a pendulum solution for actuating the flaps, and were able to increase the flutter wind speed by $50 \%$. Kwon et al. [7] introduced a passive flap control using Tuned-Mass Dampers (TMDs), achieving an increase of the flutter wind speed by $43 \%$. Finally, Aslan et al. [8] proposed a similar TMD control solution, with a different flap design, and managed to achieve a flutter wind speed increase of $10 \%$.

Even though both active and passive control strategies have proven capable of controlling bridge deck flutter, with various success rate, the passive solution has a general disadvantage: once the experimental set-up is implemented, it requires cumbersome and time-consuming procedures for introducing changes in the control parameters and thus the system response, since the components need to be modified physically, whereas active control parameters are easily adjusted through software modifications.

Moreover, all of these research groups used bridge section models endowed with a single flap on each side of the girder. We aim to extend the investigation of active flutter control to bridge section models endowed with multiple flaps, so that the potential of a physically distributed mitigation system can be studied and validated experimentally.

In this work, we present the design of a novel bridge section model, the SmartBridge, equipped with multiple actively controllable flaps, as seen in Fig. 2. Moreover, we present the first encouraging experimental results, performed 
in a wind tunnel, for validating the efficiency of our design for vibration control.

In Section II we present the design of our set-up. We introduce the experimental conditions, the support structure, and the active bridge section model itself. In Section III we present the proposed control law of the flaps, the experimental procedure, and the results obtained from actively controlling the bridge pitch. Finally, we summarize the work and give an outlook for the near future in Section IV.

\section{EXPERIMENTAL SET-UP}

We chose to design the SmartBridge as a bridge section model. A canonical, passive bridge section model is a standard tool for investigating the aeroelastic stability of a specific bridge (e.g., flutter analysis). Because of the relatively simple structure, it is well suited for testing new concepts [9]. Furthermore, all the previous experimental works presented in Section I investigating bridge flutter control using flaps [2-8] have leveraged this type of set-up.

The SmartBridge is installed in a boundary layer wind tunnel (channel dimensions: $1.5 \times 2 \times 10 \mathrm{~m}$ ) with a maximal wind speed of $16 \mathrm{~m} / \mathrm{s}$.

\section{A. Suspension, Pull-Up, and Decoupling Systems}

The SmartBridge is anchored to a suspension system, as depicted in Fig. 2. The deck is carried by two support bars that are suspended by eight springs attached to the wind tunnel structure. The design and construction of the suspension system has been significantly influenced by the suggestions given in [1].

The bridge deck position is measured with $0.1 \mathrm{~mm}$ resolution by four laser sensors (ODSL 8 from Leuze Electronics) at $200 \mathrm{~Hz}$. The sensors are placed above each corner of the deck, as seen in Fig. 2. The deck's heave and pitch are calculated from the measured distance from each sensor to the deck.

The natural frequencies in the heave Degree of Freedom (DOF), $f_{h}$, and the pitch DOF, $f_{\alpha}$ have a great impact on the system behavior, in particular the $f_{\alpha} / f_{h}$ ratio influences the flutter behavior as described in [10].

The natural frequencies in the heave and pitch DOF are in theory calculated according to the following equations:

$$
\begin{gathered}
f_{h}=\sqrt{\frac{K_{h}}{m}} \frac{1}{2 \pi}=\sqrt{\frac{8 k}{m}} \frac{1}{2 \pi} \\
f_{\alpha}=\sqrt{\frac{K_{\alpha}}{I}} \frac{1}{2 \pi}=\sqrt{\frac{K_{h} a^{2}}{I}} \frac{1}{2 \pi}=\sqrt{\frac{8 k a^{2}}{I}} \frac{1}{2 \pi}
\end{gathered}
$$

where $m$ is the mass, $I$ is the mass moment of inertia, $K_{h}$ is the stiffness in the heave DOF, $K_{\alpha}$ is the stiffness in the pitch DOF, $k$ is the stiffness of a single spring, and $a$ is the half-distance between the springs.

By adjusting the natural frequencies, we can obtain specific system responses, for instance achieve a certain wind speed where the bridge deck starts to flutter. To this purpose we have designed a system where the natural frequencies can

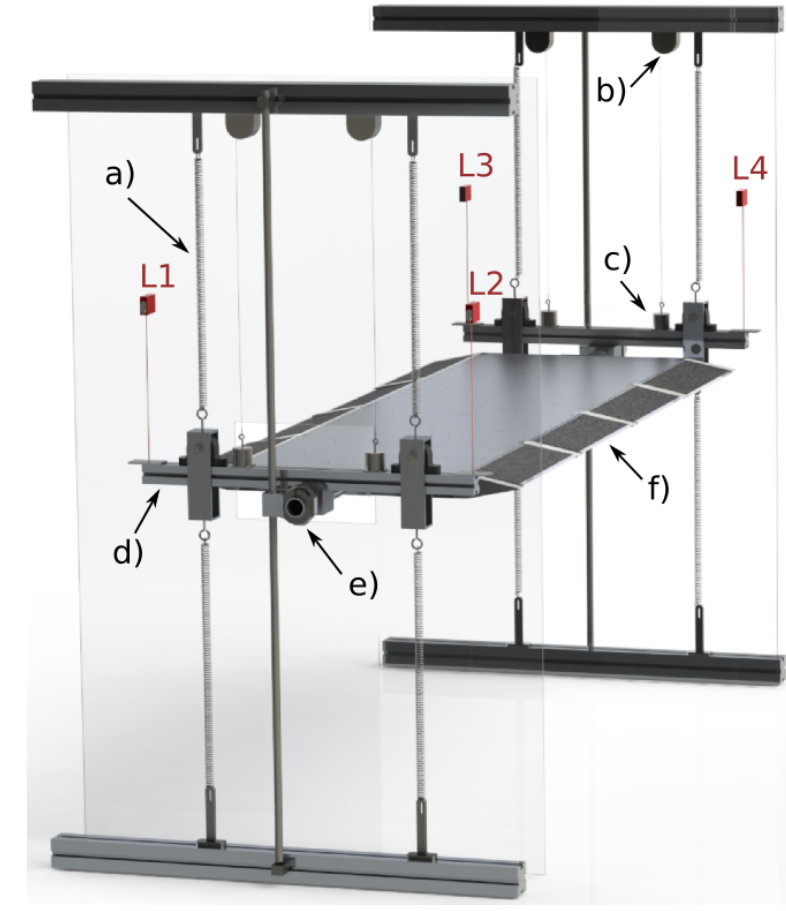

Fig. 2. The SmartBridge anchored to the suspension system. Some key elements of the set-up are highlighted in the figure: a) spring for the suspension system, b) DC motor for the pull-up system, c) electromagnet for the pull-up system, d) support bar, e) decoupling system, and f) active flap. Moreover, the four laser sensors are marked with L1-4.

easily be manipulated by changing the mass (e.g., by adding dummy masses to the support bars), the spring stiffness, and/or the distance between the springs. In the current configuration the stiffness, $k$, of one spring is $1298 \mathrm{~N} / \mathrm{m}$, the half-inter-spring-distance, $a$, is $0.29 \mathrm{~m}$, the mass, $m$, is $21.5 \mathrm{~kg}$, and the mass moment of inertia, $I$, is approximated as $0.99 \mathrm{kgm}^{2}$. Under these conditions the theoretical value for $f_{h}$ is $3.5 \mathrm{~Hz}$, according to Eq. 1. The measured frequency is $3.5 \mathrm{~Hz}$, given in Table I, and corresponds well to the theoretical value. The theoretical value for $f_{\alpha}$ is $4.7 \mathrm{~Hz}$ according to Eq. 2, and is close to the measured frequency of $4.6 \mathrm{~Hz}$. These results support the estimation of the natural frequencies according to Eq. 1 and Eq. 2.

The system parameters (e.g., natural frequencies and damping coefficients) can be identified through free vibration tests [1]. In order to perform reproduceable free vibration tests on the SmartBridge we have installed a pull-up mechanism. This mechanism is realized with four electromagnets and four DC motors, as seen in Fig. 2; the magnets can latch to the support bars and be pulled up by the motors until the deck reaches the desired set-point (the loop is closed with the bridge deck position data from the laser sensors).

In order to further facilitate the system identification of the set-up, we have designed a guiding and decoupling system that restricts the deck to the heave and pitch DOFs, as seen in Fig. 2 and Fig. 3. The heaving motion is guided with a linear bushing along a steel shaft and can be fixed by locking the linear bushing. The pitch motion is facilitated 


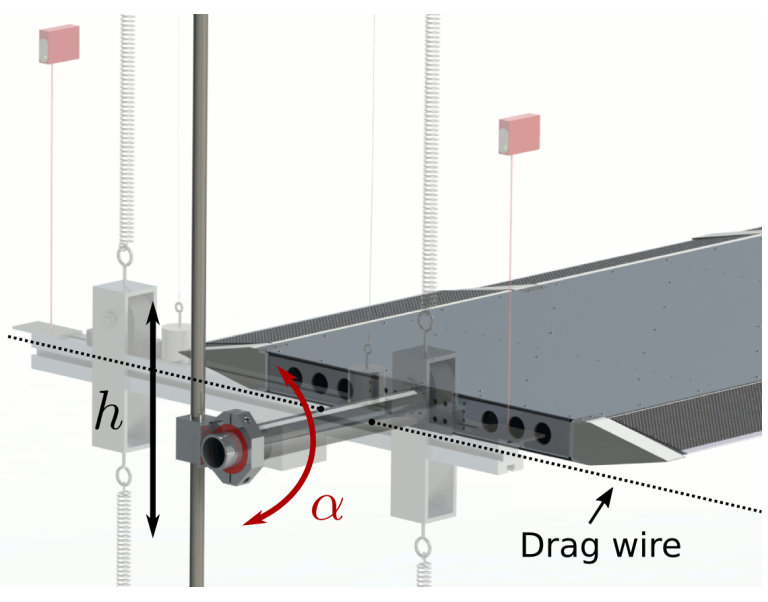

Fig. 3. The guiding system allows heave and pitch motion. Additionally, each DOF can be locked independently. The drag wires prevent motion of the deck in the horizontal DOF.

with a ball bearing and can be locked at any angle with a collar. A similar guiding system used for identifying system parameters of a canonical, passive deck, using steel shafts, linear bushings, and ball bearings were presented by Sarkar et al. [11]. Furthermore, the decoupling system is easy to add or remove depending on the type of experiment performed. In addition, drag wires [1] are permanently installed in order to prevent deck motion in the horizontal DOF even when the decoupling system is disconnected.

\section{B. Bridge Section Model}

Traditionally, passive bridge section model decks have a simple, low-cost design, yet having a high stiffness-to-weight ratio, for instance consisting of a sandwich structure with a foam core coated with aluminium plates. However, we are required to place electronics inside, draw cables and pressure tubes through the deck, which eliminates the possibility of a filled structure. Furthermore, the additional weight of the flaps put further constraints on the total mass of the SmartBridge. Therefore, we designed a novel, lightweight, hollow bridge deck in aluminium, that is inspired from airplane wing construction with its spar and rib structure, as seen in Fig. 4. The ribs and spars are permanently riveted together, while the coating panels are screwed on so that the interior remains accessible. The characteristics of the bridge section model, as

TABLE I

Measured System Parameters

\begin{tabular}{lccc}
\hline Parameter & SmartBridge & Hansen & Kobayashi \\
\hline$m[\mathrm{~kg}]$ & 21.5 & 26.6 & $\mathrm{n} / \mathrm{a}$ \\
$B^{\prime}[\mathrm{mm}]$ & 500 & 625 & 160 \\
$B[\mathrm{~mm}]$ & 740 & 938 & 240 \\
$D[\mathrm{~mm}]$ & 48 & 94 & 14 \\
$L[\mathrm{~mm}]$ & 1800 & 1480 & 450 \\
$f_{\alpha}[\mathrm{Hz}]$ & 4.6 & 1.61 & 2.7 \\
$f_{h}[\mathrm{~Hz}]$ & 3.5 & 0.83 & 2.1 \\
$B / D$ & 15.4 & 10 & 17.1 \\
$L / B$ & 2.4 & 1.6 & 1.9 \\
$f_{\alpha} / f_{h}$ & 1.3 & 1.9 & 1.3 \\
\hline
\end{tabular}

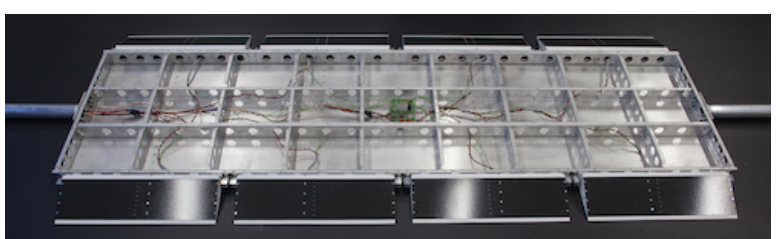

Fig. 4. Active deck, with a rib and spar structure.

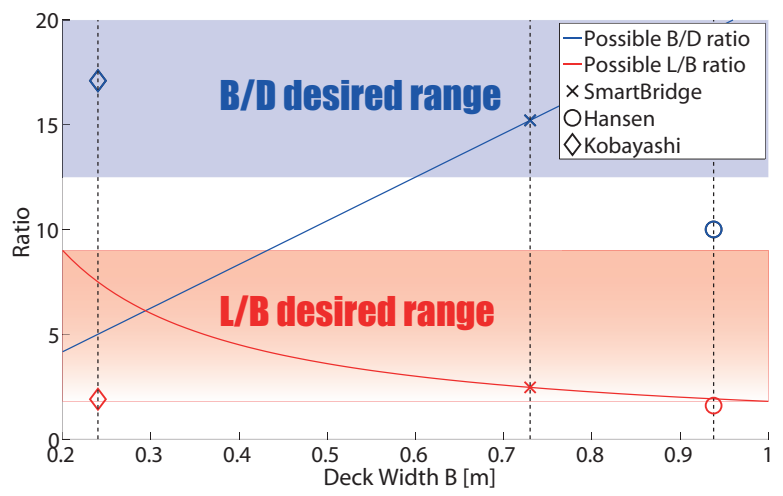

Fig. 5. Effects of changing the deck width on the $B / D$ and $L / B$ ratios.

well as corresponding parameters for the set-ups of Hansen et al. [5] and Kobayashi et al. [3], are given in Table I. The total mass, $m$, includes the mass of the deck and flaps $(14.1 \mathrm{~kg})$ and the support structure $(7.4 \mathrm{~kg})$; it corresponds to the minimum weight, which can easily be altered by adding equipment or dummy masses. The deck depth, $D$, should in principle be as thin as possible to resemble a flat plate $(D \rightarrow 0)$. In our design, the minimal depth is constrained by the size of the flap actuators. Moreover, it is common that the deck length, $L$, is as long as possible, or more precisely that the $L / B$ ratio (or aspect ratio) is high [1]. The bridge length is limited by the size of the wind tunnel in our set-up; although the distance between the channel walls is $2 \mathrm{~m}, 10$ $\mathrm{cm}$ margin per side is left to allow bridge motion as well as avoiding the turbulent wind regions close to the walls [1]. The bridge width (including flaps), $B$, is not restricted physically, although, in order to achieve a high aspect ratio, a narrower deck is preferred.

According to Matsumoto et al. [10] a $B / D$ ratio larger than 12.5 leads to a deck exhibiting coupled flutter, with increasing unstability up to a ratio of 20 . Normally, this region is avoided in a passive design; however, this is an aeroelastic instability that we desire to study and control with the flaps. As can be seen in Fig. 5 changing the deck width, $B,(L$ and $D$ are fixed values according to Table I) has contradictory desired effects of the $B / D$ and $L / B$ ratios. Finally, a compromise was found where we get a high $B / D$ ratio to obtain a deck as sensitive as possible to coupled flutter while keeping the $L / B$ ratio significantly higher (and therefore more desirable as mentioned above) than those of Hansen et al. and Kobayashi et al. 


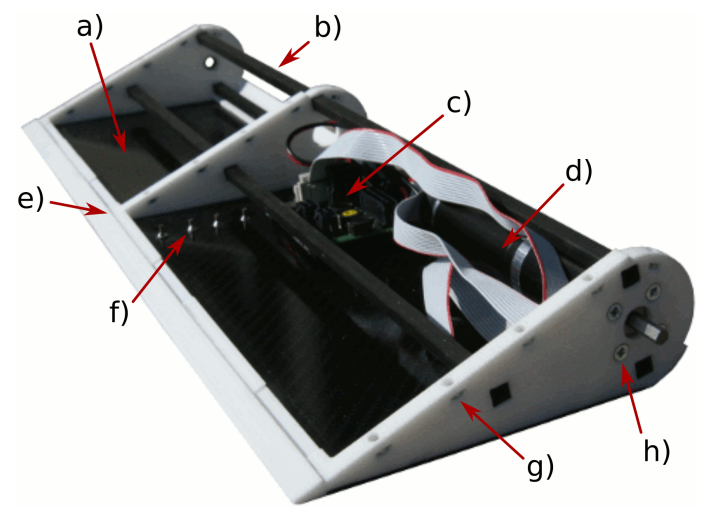

Fig. 6. A flap with the lid removed exposing some key elements of the design: a) carbon fiber sheet, b) carbon fiber rod, c) driver, d) motor, e) 3D printed frame, f) pressure tap, g) nut for attaching the lid, and h) motor anchoring to the frame.

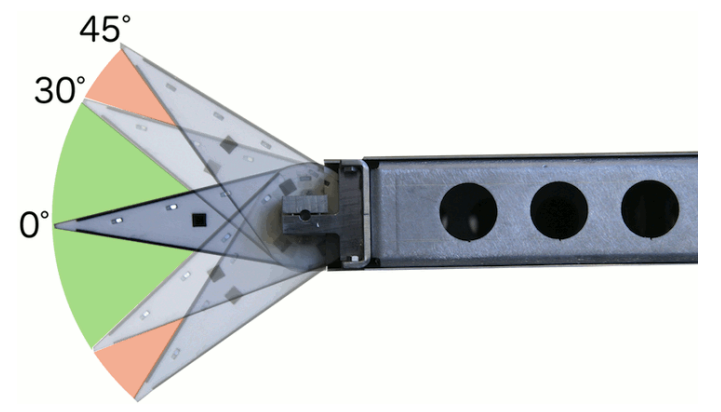

Fig. 7. The normal operating range of the flap lies within the green area. However, during the intital homing, the flap will move into the red area.

\section{Flaps and Deck Anchoring}

The current design of the flap is based on the prototype flap presented in [12], and can be found in Fig. 6. Several improvements were made to the design, the most important being the robustness of the structure. The frame of the flap is still 3D printed plastic, however the torsional rigidity was improved by increasing the wall thickness of the ribs, introducing square carbon fiber tubes through the structure, and by covering the flat sides with carbon fiber sheets (bottom side glued on and the upper side screwed on so that the interior remains accessible). Each flap is equipped with a 20 W DC motor with graphite brushes (model: 118751), gear reduction rate of 53:1 (model: 144035), optical encoder (model: 225778), and a digital positioning controller (model: 390003), all from Maxon Motor Inc. The total weight of one flap including motor, driver, cables, and screws is 550 $\mathrm{g}$. The overall shape of the flap was kept as before; however, the dimensions (length: $400 \mathrm{~mm}$, width: $139 \mathrm{~mm}$, $\max$ thickness: $48 \mathrm{~mm}$ ) were slightly adjusted to better fit the active deck requirements. Although their width is $139 \mathrm{~mm}$, the flaps on each side of the girder are only adding $2 \times 120$ $\mathrm{mm}$ to the extended bridge width $B$, since they are partially incorporated within the deck structure. This means that the effective flap width is $24 \%$ of the core deck width $B^{\prime}$ (without flaps), a value that is comparable to the $25 \%$ of Hansen et al. [5] and Kobayashi et al. [3] as can be deduced from Table I.

The flaps are attached to the deck with hinges. Furthermore, hollow, 3D printed thin shells, as seen in Fig. 9, are attached to cover the hinges and avoid turbulence at the flap fixation points. There are two main advantages of partially introducing the flap into the deck; firstly, by minimizing the physical gap between the parts, air leakage and turbulence effects are reduced; secondly, the configuration allows for a simple and reliable homing solution. At start up the flaps require homing to localize themselves. This is achieved by moving the flaps upward until the physical limit at approximately $45^{\circ}$ is reached; at this point, the motor current consumption increases above a threshold value which defines the home position; subsequently, the flap moves to an offset position that corresponds to a $0^{\circ}$ angle. Finally, a software limit is set to $\pm 30^{\circ}$, a value defining our operating range. The allowed movements of the flap are visualized by Fig. 7.

\section{Embedded system architecture}

The system overview is shown in Fig. 8. All nodes in the system, except for the laser sensors and the $\mathrm{PC}$ which are communicating via RS232, are hooked up to a CAN bus implementing a CANopen protocol (in particular CiA: DS301 and Cia: DSP-402, www.can-cia.org). The supervisor microcontroller is the master node and is communicating information to and from the whole network to the PC and a Labview ${ }^{\circledR}$ interface, from which all nodes in the network are managed. The synchronized laser sensor boards are receiving data from the laser sensors and forwarding them on the CAN bus together with a time stamp. Furthermore, the laser boards are controlling the electromagnets used for the pullup mechanism, whereas the pull-up board is solely managing the motors used for the pull-ups. The control board is reading the deck position data and calculating set-points for the flaps, thus executing the control algorithm for the flap actuation. In the current configuration the speed of the CAN bus is set to $1 \mathrm{Mbit} / \mathrm{s}$ and each laser sensor board is sending the measured distances together with a time stamp at $200 \mathrm{~Hz}$, and each motor driver is receiving a set-point and sending its actual position at $200 \mathrm{~Hz}$. Considering that each CAN data frame takes approximately $100 \mu s$ to send, a complete control loop involving 2 sensor boards and 8 motor drivers occupies the bus for $1.8 \mathrm{~ms}(100 \mu \mathrm{s} * 2+100 \mu \mathrm{s} * 2 * 8)$,

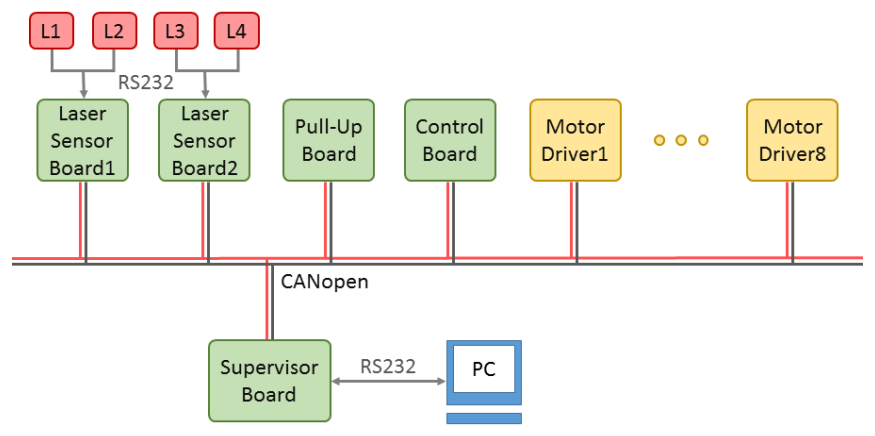

Fig. 8. A system overview of all the nodes in the network. 


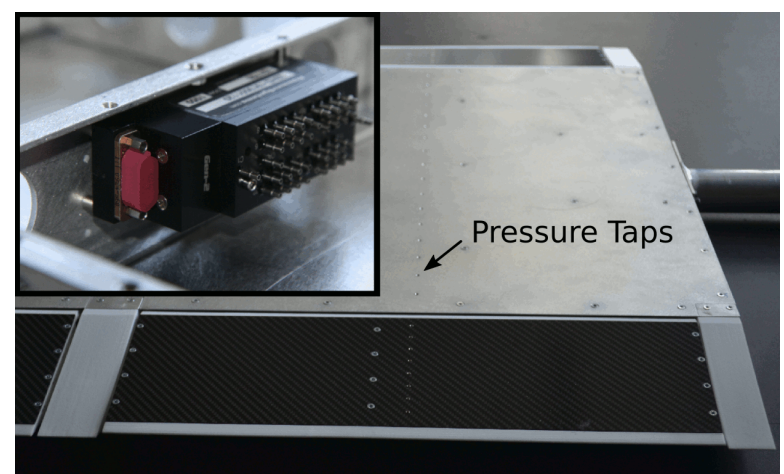

Fig. 9. The pressure taps are located along the streamline from leading edge flap, over bridge deck and to the trailing edge flap. Upper left corner: pressure sensor attachment inside deck.

i.e. a control loop at $200 \mathrm{~Hz}$ requires $36 \%$ of the $\mathrm{CAN}$ bus capacity. Since the bus is not heavily loaded, it could be further used to send additional information (e.g., state of nodes) and/or increasing the update rate for the driver nodes (e.g., at $475 \mathrm{~Hz}$ the maximal recommended bus load of $80 \%$ would be reached).

\section{E. Pressure Sensing System}

In order to better understand the underlying physical mechanisms of the active deck dynamics, we intend to analyze the pressure distribution around the deck and flaps, for which purpose we will use a 64-channel pressure sensing system. Kwon et al. [7] also measured and analyzed the pressure distribution for their passively controlled model. Moreover, the approach of using local pressure measurements to compare experimental and analytical results has also been employed for canonical passive bridge section models [13-15]. The SmartBridge has thus been designed to accommodate the pressure sensing system. The pressure is measured locally at the surface of the model, at so-called pressure taps. Pressure taps are connected to the measuring unit with tubing elements that must be kept short in order to avoid noisy measurements. Therefore, it is necessary to place the pressure measuring unit inside the deck. Furthermore, depending on the experimental purpose, it will be interesting to measure the pressure at different places. Therefore, a great number of tap locations (264) have been made throughout the bridge deck and flaps. A few tap placements as well as the pressure measurement unit are presented in Fig. 9.

\section{WIND TUNNEL EXPERIMENTS}

In order to validate the core functionalities of the SmartBridge we performed experiments in the wind tunnel. The main purpose being to ensure that the flaps are capable of real-time control of the bridge deck. Therefore we implemented a simple proportional law for the flap control using the deck pitch as input. For this purpose, we give all the flaps on the leading edge, as well as the trailing edge, the same set-point, so that they move as a single unison flap on each side of the deck. With this configuration we are using the same basic control law as Hansen et al. [5], and similar
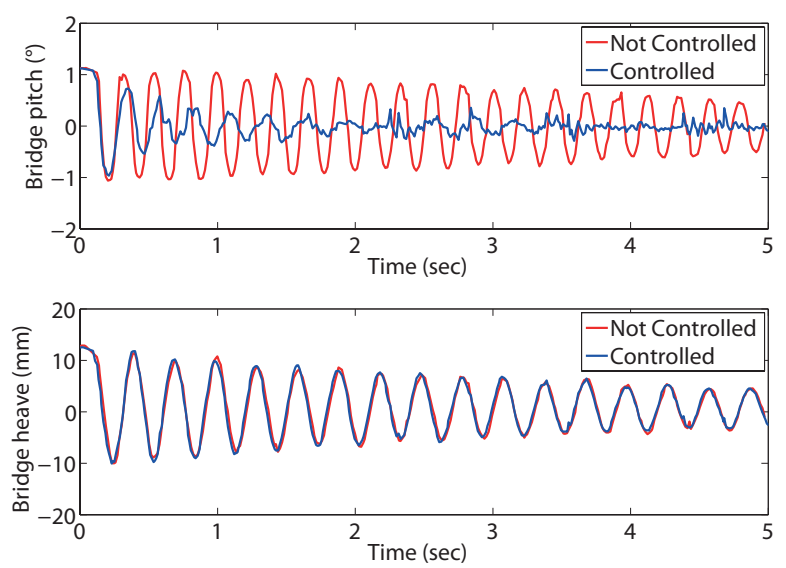

Fig. 10. Effects on the heave and pitch of the deck with and without flap control.

to Kobayashi et al. [3], who also implemented a phase shift to the control.

The bridge deck pitch can be calculated as follows:

$$
\alpha=\operatorname{atan}\left(\frac{L 2-L 1+L 4-L 3}{2 d}\right)
$$

where $L 1, L 2, L 3$, and $L 4$, are the bridge deck positions given by the laser sensors, and $d$ is the horizontal distance between a laser sensor pair. The distance, $d$, is $800 \mathrm{~mm}$ in the current set-up. However, since we operate at small pitch angles of the deck, we can use a linearized approximation of $\alpha$ for calculating on board the set-points for the leading, $\alpha_{l}$, and trailing, $\alpha_{t}$, edge flaps:

$$
\begin{aligned}
& \alpha_{l}=P_{l} *(L 2-L 1+L 4-L 3) \\
& \alpha_{t}=P_{t} *(L 2-L 1+L 4-L 3)
\end{aligned}
$$

where $P_{l}$ and $P_{t}$ are the proportional gains for the leading and trailing edge, respectively. Note that the set-points for the flaps $\alpha_{l}$ and $\alpha_{t}$ are here represented as motor quadcounts. The proportional gains were set to $P_{t}=-P_{l}$ and their numerical value was tuned manually so that the flap response was significant, yet not so sensitive that the set-points would fall outside the defined operating range. The leading edge gain was set to 10 , and trailing edge to -10 . The system was perturbed using the pull-up system. The windward side of the deck was pulled up to $20 \mathrm{~mm}$ when released, resulting in a mixed step response of the deck in the heave and pitch DOFs. The wind speed was set to $8 \mathrm{~m} / \mathrm{s}$ for the experiments.

The results of the step responses performed with flap control and without (flaps fixed at $0^{\circ}$ ) are presented in Fig. 10. It is evident that the intuitively tuned control law is already efficient: in fact it only takes the controlled deck 0.5 second to dampen the oscillations to the level achieved by the uncontrolled deck in 5 seconds. This result is comparable to the results obtained by Hansen et al. [5], where the same amplitude level was reached by their best control law three times faster than their uncontrolled case.

It is also noteworthy that the control has no visible effect on the heave vibrations, as seen in Fig. 10, a somehow expected result as this DOF is not being actively controlled. 

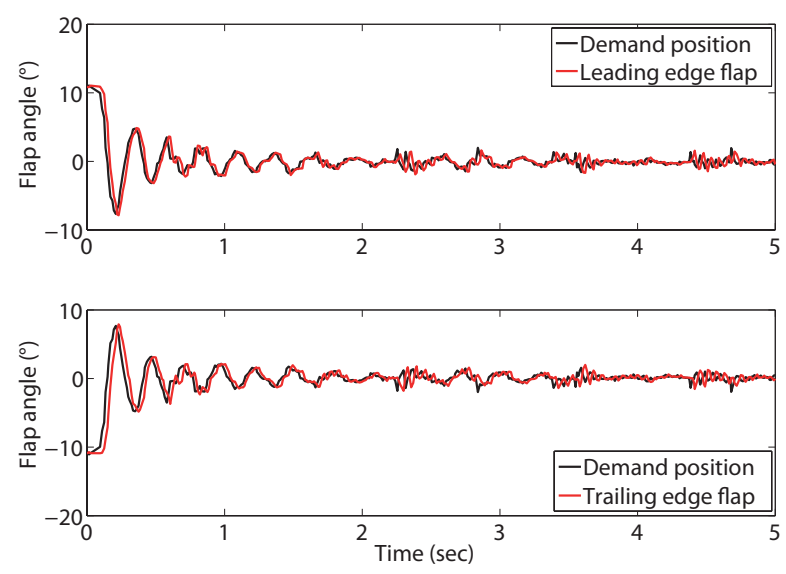

Fig. 11. Leading and trailing flap response to the bridge movements.

Furthermore, the flap response for the controlled deck is presented in Fig. 11. For the sake of clarity we only present the result of one of the flaps on the leading and trailing edges, however all four flaps on each side are moving with similar trajectories. The set-points were given in motor quadcounts, calculated according to Eqs. 4 and 5, but are here converted to degrees. It is clear that the flaps are able to cope with the fast dynamics of the bridge deck; although there is a small lag, the flaps are able to follow the demand trajectories well.

\section{Conclusions}

In this paper, we have presented a novel bridge section model equipped with actively controlled flaps; a dedicated suspension system, a pull-up mechanism, and a decoupling system complete the overall set-up. The design of the bridge deck allows for easy access to electronics, cables, and tubing. Furthermore, the design is modular and changeable, allowing modifications of system parameters such as natural frequencies, and providing a flexible platform for conducting pressure measurements.

We have experimentally validated the core functionalities of the set-up by succesfully controlling and stabilizing the pitch of the bridge deck. Although the control algorithm was simple and manually tuned, the efficiency of the actively controlled flap system was significant and comparable to previous results acheived by other researchers.

The next step will be to leverage all the features of the system presented here, such as the decoupling system and pressure measurements, so that we can properly model the bridge dynamics and further investigate more sophisticated control strategies.

\section{REFERENCES}

[1] A. Larsen, "Aerodynamics of Large Bridges", Taylor \& Francis, 1992.

[2] H. Kobayashi and H. Nagaoka, "Active Control of Flutter of a Suspension Bridge", Journal of Wind Engineering and Industrial Aerodynamics, vol. 41, pp. 143-151, 1992.
[3] H. Kobayashi, R. Ogawa, and S. Taniguchi, "Active Flutter Control of a Bridge Deck by Ailerons", in Proceedings of Second World Conference on Structural Control, Kyoto, Japan, 1998.

[4] H. I. Hansen and P. Thoft-Christensen, "Active Vibration Control of Long Suspension Bridges", PhD Thesis, Dept. of Building Technology and Structural Engineering, Aalborg University, 1998.

[5] H. I. Hansen, P. Thoft-Christensen, P. A. Mendes and F. A. Branco, "Wind-Tunnel Tests of a Bridge Model with Active Vibration Control", Structural Engineering International, vol. 10, pp. 249-253, November, 2000.

[6] K. Wilde, Y. Fujino, and T. Kawakami, "Analytical and Experimental Study on Passive Aerodynamic Control of Flutter of a Bridge Deck", Journal of Wind Engineering and Industrial Aerodynamics, vol. 80, pp. 105-119, 1999.

[7] S. D. Kwon, M. S. Sungmoon Jung and S. P. Chang, "A New Passive Aerodynamic Control Method for Bridge Flutter", Journal of Wind Engineering and Industrial Aerodynamics, vol. 86, pp. 187-202, 2000.

[8] H. Aslan and U. Starossek, "Passive Control of Bridge Deck Flutter Using Tuned Mass Dampers and Control Surfaces", presented at the 7th European Conference on Structural Dynamics, Southampton, UK, 2008.

[9] E. Simiu and R. H. Scanlan, 'Wind Effects on Structures: Fundamentals and Applications Design", John Wiley \& Sons, 1996.

[10] M. Matsumoto and K. Mizuno and K. Okubo and Y. Ito, "Torsional Flutter and Branch Characteristics for 2-D Rectangular Cylinders", Journal of Fluids and Structures, vol. 21, pp. 597-608, 2005.

[11] P. P. Sarkar, A. G. Chowdhury and T. B. Gardner, "A Novel Elastic Suspension System for Wind Tunnel Section Model Studies", Journal of Wind Engineering and Industrial Aerodynamics, vol. 92, pp. 23-40, 2004.

[12] M. Boberg, G. Feltrin, and A. Martinoli, "Model and Control of a Flap System Mitigating Wind Impact on Structures", in 2014 IEEE International Conference on Robotics and Automation (ICRA), Hong Kong, China, 2014, pp. 264-269.

[13] B. Cao and P. P. Sarkar, "Identification of Rational Functions by Forced Vibration Method for Time-Domain Analysis of Flexible Structures", in The Fifth International Symposium on Computational Wind Engineering, Chapel Hill, US, 2010.

[14] A. Sarkic, R. Fisch, R. Hoeffer and K. U. Bletzinger, "Bridge Flutter Derivatives Based on Computed Validated Pressure Fields", Journal of Wind Engineering and Industrial Aerodynamics, vol. 104-106, pp. 141-151, 2012.

[15] T. Argentini, D. Rocchi, S. Muggiasca and A. Zasso, "Cross-Sectional Distributions Versus Integrated Coefficients of Flutter Derivatives and Aerodynamic Admittances Identified with Surface Pressure Measurements", Journal of Wind Engineering and Industrial Aerodynamics, vol. 104-106, pp. 152-158, 2012. 